\title{
Structural, Chemical, and Local Properties of Layered Metal Chalcophosphate Systems
}

\author{
Matthew Cheng, Roberto dos Reis, Daniel Chica, Mercouri Kanatzidis and Vinayak Dravid
}

Northwestern University, Evanston, Illinois, United States

Due to the variety of unique properties that arise as a function of dimensional confinement, two dimensional materials have received significant interest from the research community in recent years. The atomically thin family of materials include Dirac semimetals (graphene), direct bandgap semiconductors (molybdenum disulfide), ferromagnetic materials (chromium triiodide) and many others [1,2]. While these one-component and two-component systems have enabled a variety of advances, scientists have made efforts to more heavily investigate unique multicomponent layered systems in an effort to achieve other exotic phenomena. These include the metal chalcophosphates (MCPs) family of van der Waals (vdW) materials which were first discovered in the 1980s. Recent studies into the metal chalcophosphates (MCPs), have brought to light a multitude of properties that make them suitable for applications including energy storage [3], neutron detection [4], and non-volatile memory storage applications [5].

The MCPs adopt either a $\mathrm{M}_{2}{ }^{2+} \mathrm{P}_{2} \mathrm{X}_{6}$ or $\mathrm{M}^{1+} \mathrm{M}^{3+} \mathrm{P}_{2} \mathrm{X}_{6}$ stoichiometry and are comprised of a layer of transition metal cations sandwiched between two layers of chalcogen $(\mathrm{S}, \mathrm{Se})$ anions with a phosphorous dimer occupying every third octahedral cation site. In the mixed cation stoichiometry of the MCPs, $\mathrm{M}^{1+} \mathrm{M}^{3+} \mathrm{P}_{2} \mathrm{X}_{6}$ particular attention is paid to the $\mathrm{Cu}$ containing chalcophosphates which have been shown to exhibit ferroelectric (FE) properties due to a second order Jahn-Teller distortion at the $\mathrm{Cu}^{1+}$ site [6]. The second order Jahn-Teller distortion at the $\mathrm{Cu}^{1+}$ site brings about a spontaneous net polarization in the out-of-plane direction that directly influences the FE domain structure of these materials. It is therefore, of utmost importance to carefully assess and understand the polarization properties of these materials in the out of plane direction for future integration into memory storage devices. Scanning transmission electron microscopy (STEM) and piezoresponse force microscopy (PFM) are optimal techniques to observe and study the ferroelectric domains that are present in these material systems. Unlike bulk measurements, both techniques offer incredible spatial resolution on the order of sub-angstroms to nanometers which offers a route to probe the underlying structure of these materials in order to better understand the mechanisms that influence the FE domain structures.

In this study, we report on the structural characterization of $\mathrm{CuInP}_{2} \mathrm{~S}_{6}$ and the spatial variation in polarization that are present due to the spontaneous polarization within the layers of this vdW material. A cross-sectional TEM sample was prepared using focused ion beam (FIB) in order to view the sample in the in-plane direction. High resolution transmission electron microscopy (HRTEM) images were taken along the - zone axis with accompanying selected area electron diffraction (SAED) patterns in order to visualize distortions or changes in the crystal lattice and to observe defects that are present (Figure 1).

The FE domains are imaged using Dual Amplitude Resonance Tracking (DART) enhanced PFM (Figure 2). The natural domains that form in the $\mathrm{CuInP}_{2} \mathrm{~S}_{6}$ system can be seen in the amplitude (top image) and phase (middle image) map. Note the bright regions in the phase map versus the dark regions. There is a nearly perfect 180 degree out of phase response between the two regions. The corresponding amplitude map also indicates contrast in the same regions as the phase map. 
Ongoing efforts include creating cross sectional samples of $\mathrm{CuInP}_{2} \mathrm{~S}_{6}$ in order to conduct lateral PFM measurements to study the domains along the stacked layers of $\mathrm{CuInP}_{2} \mathrm{~S}_{6}$ as well as conducting detailed structural and chemical analysis of these unusual structures with aberration corrected STEM. We believe that these metal chalcophosphates represent an interesting emerging class of two-dimensional materials that exhibits promising properties that may serve as a platform for additional opportunities in creating artificial stacks and synthetic architectures. [7]

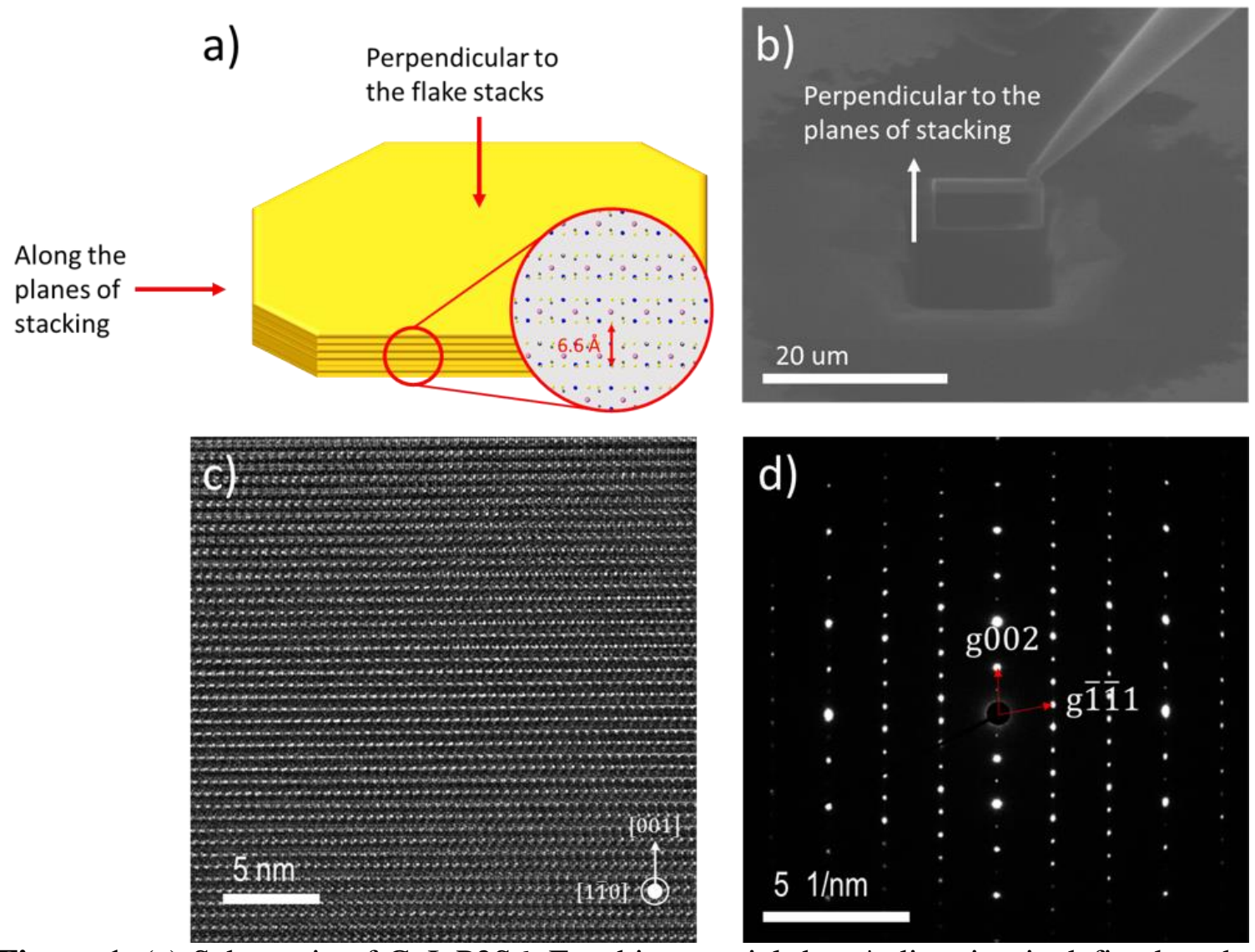

Figure 1. (a) Schematic of CuInP2S6. For this material the $\mathrm{c}^{*}$-direction is defined as the perpendicular direction as shown in the atomic model. (b) Focused Ion Beam (FIB) lift out of TEM cross sectional sample viewed along the [ $1-10]$ - zone axis and with the direction indicating the planes of stacking. (c) High Resolution Transmission Electron Microscope (HRTEM) image of CuInP2S6 viewed along [1 -1 0] - zone axis. In this case, [ $\left.\begin{array}{lll}1 & -1 & 0\end{array}\right]$ - direction is "along the planes of stacking" and the [ $\left.\begin{array}{lll}0 & 0 & 1\end{array}\right]$ - direction is perpendicular to the planes of stacking. (d) Selected area electron diffraction (SAED) pattern along the [1 -1 0] - zone axis. 


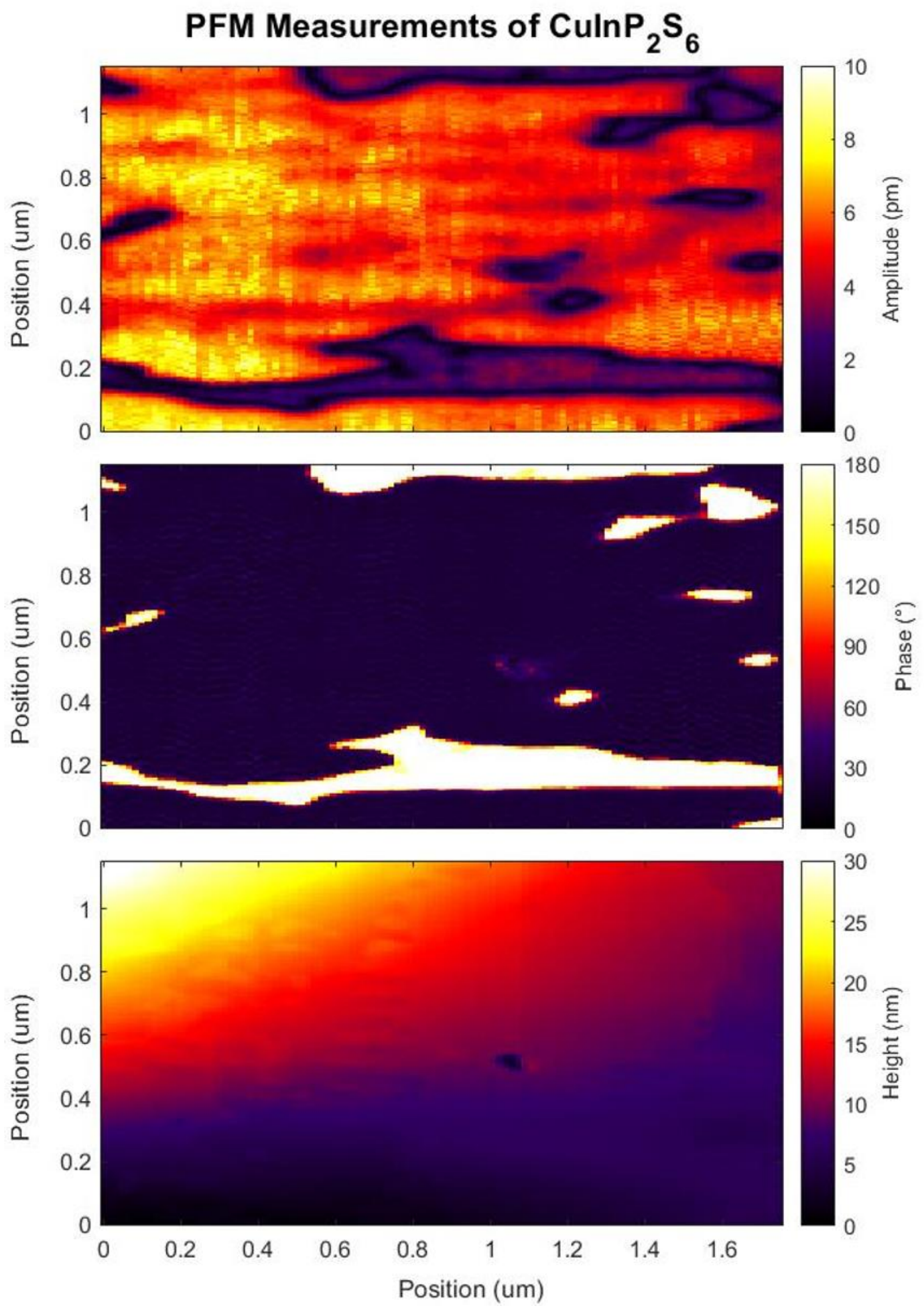

Figure 2. Piezoresponse Force Microscopy (PFM) maps on a sample of CuInP2S6. (Top) Amplitude response map. (Middle) Phase response map. (Bottom) Topographical map. Measurements taken with Asylum Research Cypher AFM and Simple Harmonic Oscillator (SHO) model applied.

References 
[1] Geng, D. et al. Adv. Funct. Mater. 2018, 30, 1800865.

[2] Li, T. et al. Nat. Mat. 18, 1303-1308 (2019).

[3] Liang, Q et al. Small Methods 2017, 1, 1700304.

[4] Chica, D. et al. Nature 577, 346-349 (2020).

[5] Liu, F. et al. Nat. Commun. 7, 12357 (2016)

[6] Maisonneuve, V. Journal of Solid State Chemistry 116, 208-210 (1995).

[7] This material is based upon work supported by the NSF (DMR-1929356) and made use of the EPIC and SPID facilities of Northwestern University's NUANCE Center, which has received support from the Soft and Hybrid Nanotechnology Experimental (SHyNE) Resource (NSF ECCS-1542205); the MRSEC program (NSF DMR-1720319) at the Materials Research Center; the International Institute for Nanotechnology (IIN); the Keck Foundation; and the State of Illinois, through the IIN. M.C. gratefully acknowledges support from the NSF GRFP and the Cabell Fellowship. 